\title{
An integrative review of the current evidence on the relationship between hand hygiene interventions and the incidence of health care-associated infections
}

Chantal Backman, RN, BScN, MHA, ${ }^{a}$ Dick E. Zoutman, MD, FRCPC, ${ }^{\text {b }}$ and Patricia Beryl Marck, RN, BScN, MN, PhD ${ }^{\mathrm{c}}$ Ottawa, Kingston, and Edmonton, Canada

\begin{abstract}
Background: The objectives of this integrative review were to critically examine the overall state of the current evidence on the relationship between hand hygiene interventions and the incidence of health care-associated infections (HCAIs) in acute care and long-term care settings, and offer recommendations for future directions in the field based on our findings.

Methods: We searched for original research and reviews of research published between January 1, 1996, and July 31, 2006. Studies were identified through the electronic databases Medline, CINAHL, EMBASE, PUBMED, the Cochrane Library, and through expert consultation. Our comprehensive search strategy included all English articles for which hand hygiene or handwashing-related terms were combined with HCAIs. All studies that investigated a relationship between hand hygiene practices and HCAIs in acute care facilities were considered. These hand hygiene practices included the initiation of multimodal hand hygiene initiatives, the introduction of alcohol sanitizers, the implementation or changes of the infection control practices or infection control policies, and other organizational interventions. Studies only examining hand hygiene compliance, efficacy of alcohol hand gels, plain soap, and antimicrobial soap in reducing bacteria count recovered from hands were excluded.

Results: Of the 1120 articles retrieved, 35 publications, including 4 reviews of research discussed at the outset of this article, met our inclusion criteria. The remaining 31 eligible original studies included $18(58.07 \%)$ before and after studies without control groups, $4(12.90 \%)$ before and after studies with a control group, $3(9.68 \%)$ cohort studies with no control group, $4(12.90 \%)$ cohort studies with a control group, and $2(6.45 \%)$ randomized trials. Over $50 \%$ (16 or 31 ) of the studies were conducted in the U.S. Two independent reviewers conducted independent evaluations of all eligible studies, critiquing and scoring each study using a rating scale for examining the fatal flaws of quasi-experimental and before and after studies.

Conclusions: There is a lack of rigorous evidence linking specific hand hygiene interventions with the prevention of HCAIs. The varied nature of the interventions used and the diverse factors affecting the acquisition of HCAIs make it difficult to show the specific effect of hand hygiene alone. The most frequent methodologies currently used in this research area are before and after observational studies without a control comparison group. Based on these findings, we recommend that researchers used a modified version of Larson's 2005 criteria to guide the design and conduct of future before and after observational studies in this area. We also argue that as we accumulate stronger evidence of which interventions are most effective, we need to develop additional research approaches to study how organizations succeed and fail in fostering the uptake of evidence-based hand hygiene interventions. (Am J Infect Control 2008;36:333-48.)
\end{abstract}

Health care-associated infections (HCAIs) are one of the most frequent and severe complications of hospitalization and the fourth leading cause of death in

From the Faculty of Nursing, ${ }^{a}$ University of Alberta, Edmonton, Alberta and Canadian Patient Safety Institute, Ottawa, Ontario; Department of Pathology and Molecular Medicine, ${ }^{b}$ Queen's University and Infection Control Service, Kingston General Hospital, Kingston, Ontario; and Faculty of Nursing and John Dossetor Health Ethics Centre, ${ }^{c}$ University of Alberta and Royal Alexandra Hospital Clinical Research Unit, Capital Health, Edmonton, Alberta, Canada.

Address correspondence to Chantal Backman, RN, BScN, MHA, Canadian Patient Safety Institute, 100-1730 St. Laurent Blvd, Ottawa, Ontario KIG 5LI, Canada. E-mail: cbackman@cpsi-icsp.ca.

$0196-6553 / \$ 34.00$

Copyright (c) 2008 by the Association for Professionals in Infection Control and Epidemiology, Inc.

doi: 10.1016/j.ajic.2007.08.007
Canada. ${ }^{1}$ One in 9 patients contract an infection while in a hospital, and an estimated 8000 patients die directly or indirectly each year from HCAIs. ${ }^{2}$ According to 1995 US data, "between $5 \%$ and $10 \%$ of patients admitted to acute care hospitals acquire 1 or more infections, and the risks have steadily increased during recent decades." 3

Although many factors contribute to the development of HCAIs, the consistent practice of adequate hand hygiene, either by washing the hands with soap and water or disinfecting them with an antiseptic solution, ${ }^{4}$ is considered to be "the single most important intervention to prevent nosocomial infections." 5 However, compliance with hand hygiene among health care professionals remains relatively low. Some of the reasons identified by Pittet for poor compliance include skin irritation, inaccessibility to handwashing 
supplies, lack of adequate hand hygiene facilities, health care workers "not thinking about it," high workloads and inadequate time, busy schedule, time-consuming, hands don't appear soiled, poor location of sinks, and lack of education about hand hygiene in the curricula. ${ }^{6}$ Suresh and Cahill have also noted several issues with the structural layout of hospital wards in their ergonomic assessment of 4 hand hygiene resources (sinks, alcohol sanitizer dispensers, gloves, and garbage bins), which are essential to creating a "user-friendly" hospital environment for the reliable practice of effective hand hygiene. ${ }^{7}$ In turn, the complexities of designing, implementing, and evaluating hand hygiene interventions and other infection control programs are critically shaped by the adequacy of health system resources for infection surveillance and control. ${ }^{2}$ Nevertheless, the Canadian Patient Safety Institute has signalled that reducing preventable HCAIs is a priority in modern health care. Other countries around the world, including the United Kingdom, Australia, and Switzerland, are in agreement that a multimodal strategy to hand hygiene is necessary to reduce the occurrence of HCAIs; however, the relative contribution of each intervention is difficult to measure.

To date, 4 reviews of research have examined the evidence of a causal link between hand hygiene and risk of infection. In 1988, Larson ${ }^{8}$ reviewed the published literature between 1879 and 1986 and found that the majority of publications were either evaluations of particular products or antiseptics $(50.8 \%)$ or review articles $(29.1 \%)$. Approximately $11 \%$ of the articles were concerned with behavioral aspects of hand hygiene, and only 14 (3.3\%) of the studies reviewed specifically linked hand hygiene with the incidence of infection. Even though there were very few prospective studies assessing the causal link between hand hygiene and the incidence of HCAIs, the collective evidence from this initial review was judged to be consistent with the hypothesis that adequate hand hygiene is associated with a decrease incidence of HCAIs. In a subsequent review of research published between 1977 and 1998, Larson ${ }^{9}$ found a temporal relationship between improved hand hygiene and reduced rates of HCAI but noted that the causal link in the majority of these studies was weak.

In a third review, Larson ${ }^{10}$ examined the published research for a 1-year period (2004) that assessed the evidence linking hand hygiene interventions with a reduction in HCAIs. Most studies $(69.2 \%)$ reported a statistically significant reduction in infection rates as a result of the implementation of various infection control interventions. Based on these findings, Larson noted the importance of senior leader and middle management commitment to administrative and system changes that are required to successfully implement and sustain hand hygiene best practices. In a fourth review of research published in 2005 , Silvestri et $\mathrm{al}^{11}$ analyzed the effectiveness of hand hygiene on infection rates in the intensive care unit. Those authors' results argued that hand hygiene alone can only reduce the level of contamination by $40 \%$ at most and cannot be expected to eliminate completely the incidence of HCAIs. Silvestri et al recommended that a multicenter, randomized controlled trial on the effect of hand hygiene on all infections was needed to support the statement from the Handwashing Liaison Group that adequate hand hygiene is a practice that can demonstrate big effects.

To build on these previous reviews of research, we searched for original research and reviews of research published between January 1, 1996, and July 31, 2006, to (1) critically examine the overall state of current evidence on the relationship between hand hygiene interventions and the incidence of HCAIs in acute care and long-term care settings and (2) offer recommendations for the design and conduct of future research, research syntheses, and policy in the field.

Our primary goal in conducting this critique was to identify specific organizational hand hygiene interventions that were clearly associated with significant reductions in the incidence of HCAIs in acute care and longterm care settings. We also sought to do as Pawson et al recommend, which is to reconsider how we conduct and learn from organizational intervention research in modern, complex health systems to strengthen practice and improve the outcomes of care. ${ }^{12}$

\section{METHODS}

\section{Search strategy}

Studies, published between January 1, 1996, and July 31, 2006, were identified through the electronic databases of Medline, CINAHL, EMBASE, PUBMED, The Cochrane Library, and expert consultation. We conducted a comprehensive search strategy that included all English articles with the MESH headings handwashing, the term hand combined with related terms such as disinfection, antisepsis, disinfectants, antiinfective agents, local, antiviral agents, soaps, detergents, ethanol or alcohols and articles with a title that included the following terms: (hand\$ adj5 wash\$), handwashing\$, hand hygiene, (hand\$ adj5 [sanitis or disinfects or decontaminats or gel\$]). These terms were combined with the terms cross infection and infection control and other terms such as nonsocomial, nosocomial\$, (reduc\$ adj3 spread\$), disease transmission, and (health care associated or health care associated or hospital acquired). Further details of the search strategy are available in Table 1. 
Table I. Search strategy, review period: January I, 1996- July 3I, 2006

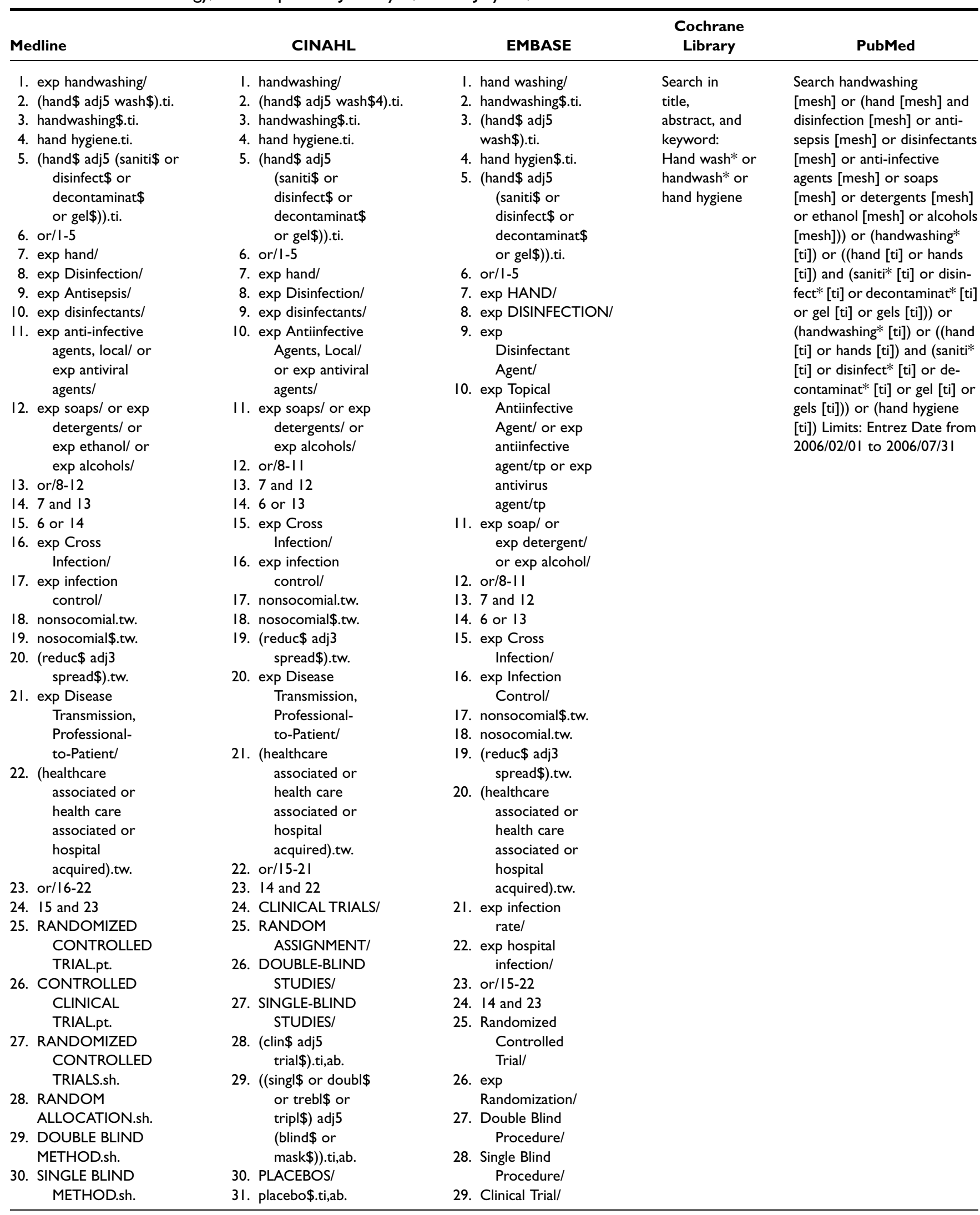


Table I. Continued

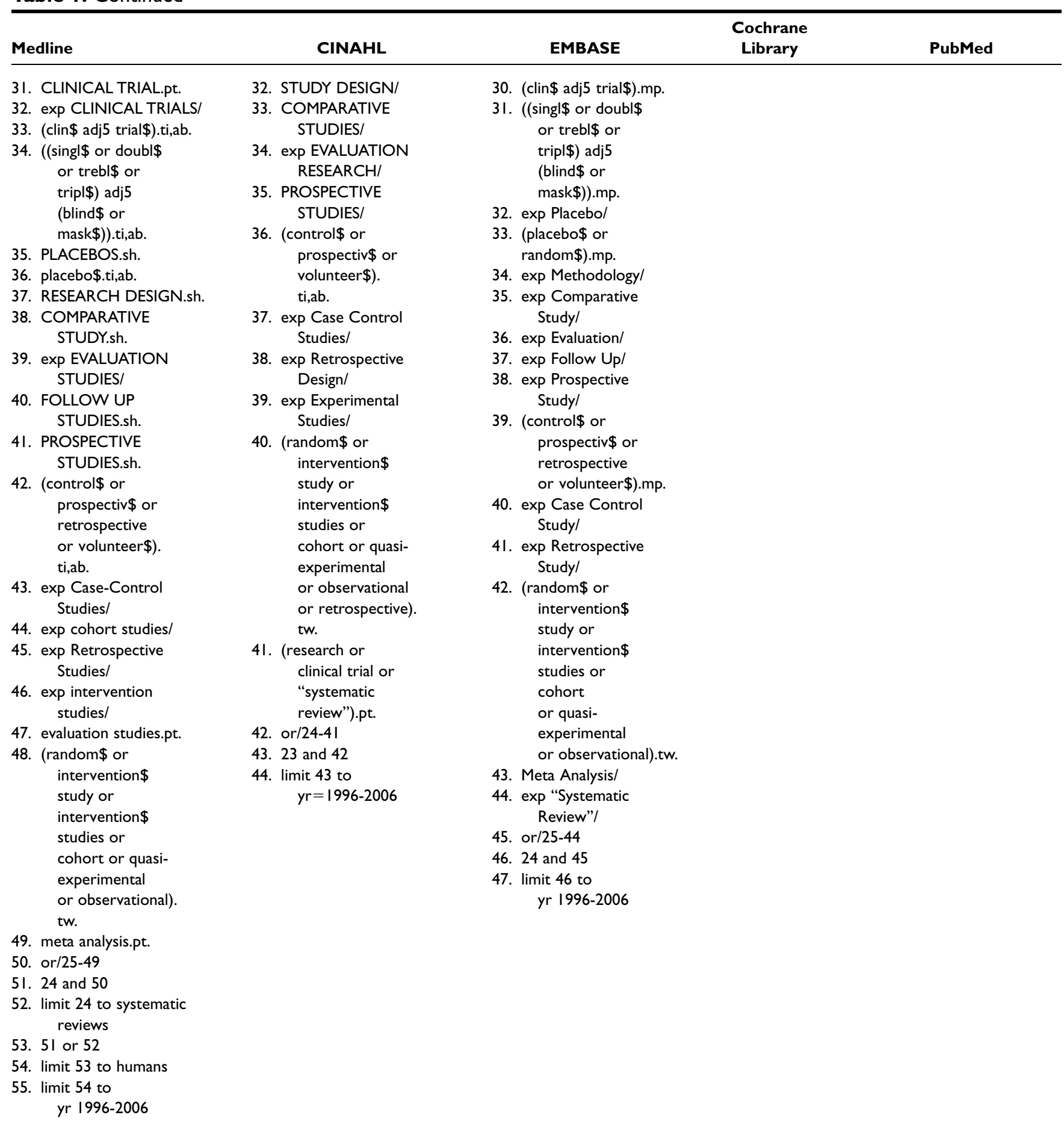

\section{Inclusion criteria}

All studies that investigated a relationship between hand hygiene interventions and HCAIs in acute care and long-term care facilities were considered. Studies that only examined the rate of hand hygiene compliance or the efficacy of alcohol hand gels, plain soap, or antimicrobial soap in reducing bacteria count recovered from hands were excluded. A complete list of the inclusion and exclusion criteria is outlined in Table 2. We defined a hand hygiene intervention (HHI) as any program of hand sanitation that an organization implements to promote compliance with desired standards of hand cleanliness. We defined HCAIs, in accordance 
Table 2. Inclusion and exclusion criteria

\begin{tabular}{|c|c|c|}
\hline & Inclusion & Exclusion \\
\hline Period & $\begin{array}{l}\text { January I, I996-July 3I, } 2006 \text { (I0 yr) } \\
\text { English only }\end{array}$ & \\
\hline Population/setting & $\begin{array}{l}\text { All health care settings (where care is received in } \\
\text { facility, 24-hr coverage by RN care) } \\
\text { Developed countries only }\end{array}$ & $\begin{array}{l}\text { All other settings (community, daycare, schools, } \\
\text { and others) } \\
\text { Developing countries }\end{array}$ \\
\hline Intervention & All hand hygiene interventions & $\begin{array}{l}\text { Glove use, masks, gowns } \\
\text { Hospital design/architecture } \\
\text { Dental products } \\
\text { Antibiotics or drug therapy } \\
\text { Artificial nails } \\
\text { Food handling } \\
\text { Isolation precautions } \\
\text { Surveillance }\end{array}$ \\
\hline Outcome & All health care-associated infections & \\
\hline \multirow[t]{14}{*}{ Study design } & Experimental studies & $\begin{array}{l}\text { Reviews that did not clearly define the parameters } \\
\text { of their search strategy, including inclusive dates of the } \\
\text { review, databases searched, and search terms used }\end{array}$ \\
\hline & Randomized controlled trial & Expert opinion or consensus \\
\hline & Experimental study without randomization & Editorial letter \\
\hline & Observational study with control group & Guideline implementation \\
\hline & Cohort study & Commentary \\
\hline & Case control study & Outbreak reports \\
\hline & Observational study without control groups & \\
\hline & Cross-sectional study & \\
\hline & Before-and after study & \\
\hline & Cases series & \\
\hline & Reviews of research (only if they clearly defined the & \\
\hline & parameters of their search strategy, including inclusive & \\
\hline & dates of the review, databases searched, and search terms & \\
\hline & used) & \\
\hline
\end{tabular}

with the Centers for Disease Control and Prevention, as infections that occur 72 hours or more after admission that are not in incubation prior to admission. ${ }^{13}$ The HHI at issue could be as simple as making cleaning agents and washing facilities readily available or as complex as a multimodal quality improvement program that is designed to increase the rate of hand hygiene compliance through education, incentives, facility design, and other measures within a given care environment. The interventions we reviewed included the initiation of educational programs or campaigns, multimodal hand hygiene initiatives, introduction of alcohol sanitizers, hand hygiene performance improvement, and implementation of changes in the infection control practices.

Of the 1120 papers retrieved, there were 35 studies that met the inclusion criteria. Two reviewers (C.B. and D.Z.) conducted independent evaluations of all eligible studies, critiquing and scoring each study using a rating scale designed to identify the fatal flaws of quasiexperimental and before and after studies (Table 3). ${ }^{10}$ Following the initial round of independent reviews, all 3 coauthors discussed the findings and gaps between the scores of the first and second reviewer to ascertain the rationale for any disparate scores between reviewers. Final scores were then determined and assigned to each study based on group consensus.

\section{RESULTS}

\section{Characteristics of the studies}

Thirty-five publications, including 4 reviews of research discussed at the outset of this article, met our inclusion criteria. The remaining 31 eligible original studies included the following: 18 (58.07\%) before and after studies without control groups, 4 (12.90\%) before and after studies with a control group, 3 $(9.68 \%)$ cohort studies with no control group, 4 $(12.90 \%)$ cohort studies with a control group, and 2 $(6.45 \%)$ randomized trials.

By type of intervention, the 31 studies were categorized as follows: 7 (22.58\%) initiations of a multimodal hand hygiene initiative, $11(35.48 \%)$ introductions of a hand hygiene product, $8(25.81 \%)$ implementations or modifications of infection control practices, 2 (6.45\%) implementation of infection control policies, and 3 $(9.68 \%)$ other interventions (top level administration intervention, electronic monitoring, or survey on 
Table 3. Fatal flaws of quasiexperimental and before and after studies ${ }^{10}$

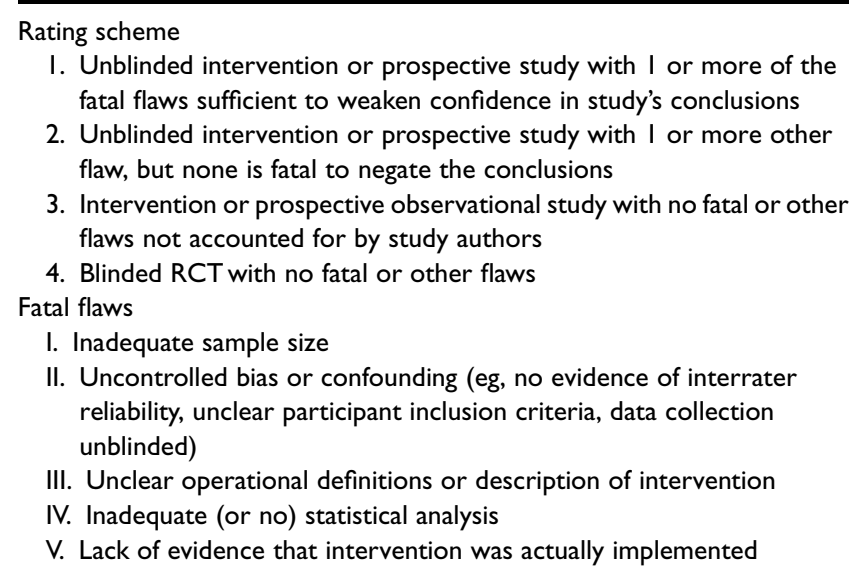

current practices). Multiple types of HCAIs ( 2 or more) were examined in 26 studies $(74.28 \%)$. Other studies observed the rates of a single infection such as methicillin-resistant Staphylococcus aureus (MRSA), vancomycin-resistant Enterococcus (VRE), bloodstream infections, rotavirus, surgical site infections, and Clostridium difficile. Most of the studies, 18 of 31 (58.06\%), reported a significant reduction in HCAIs (Table 4). Only 5 studies reported a power calculation.

Using Larson's 2005 scoring tool, ${ }^{10}$ most of the studies $(67.7 \%)$ were rated with a score of 1 or 2 , indicating that methodologic flaws were found. We assigned a score of 1 to 11 of the studies ( $35.48 \%)$, indicating that there were 1 or more fatal flaws found in the study. Ten of the studies (32.26\%) received a score of 2 because they contained 1 or more other (nonfatal) flaw but no fatal flaws. We assigned a score of 3 to 9 intervention or observational studies $(29.03 \%)$ with no fatal or other flaws. Only 1 of the studies $(3.23 \%$ ) received a score of 4 , indicating a randomized study with no fatal or other flaws. The 3 most common flaws identified in the studies were the presence of confounding factors not considered or identified (8 studies), inadequate statistical analysis (6 studies), and inadequate sample size (5 studies). Seven studies (22.58\%) had more than 2 fatal flaws. In the next section, we elaborate on our findings for each category of studies by type of intervention.

\section{Category 1. Multimodal hand hygiene initiatives}

Seven studies $(22.58 \%)$ initiated a multimodal hand hygiene initiative to study its impact on the reduction of HCAIs. Six of these studies were before and after studies with no control group, ${ }^{14-19}$ and 1 was a cohort study. ${ }^{20}$ Of these studies, two ${ }^{14,18}$ reported a significant reduction in HCAI rates $(P=.04$ and $P=.003$, respectively) and two ${ }^{15,19}$ reported no significant reductions in overall HCAI rates. Two studies ${ }^{14,20}$ reported a significant reduction $(P<.001$ and $P=.01$, respectively) in MRSA rates, and 1 study $^{17}$ reported a decrease in MRSA and VRE rates followed by an increased trend in MRSA and VRE rates after year one, but the results were not significant. Finally, a decrease in the rotavirus rate was reported in the results of one other study. ${ }^{16}$

Two of the studies ${ }^{14,20}$ had confounding variables, which made it difficult to determine which aspects of the multimodal approach actually accounted for the reduced rates of HCAIs reported. Four of the 7 studies $^{16-19}$ had no fatal or other flaws.

\section{Category 2. Hand hygiene products}

Eleven studies (35.48\%) introduced a hand hygiene product to study its impact on the reduction of HCAIs. In 5 of these studies, one before and after design with no control group ${ }^{21}$ and 4 cohort studies with control groups $^{22-25}$ compared handwashing with the introduction of an alcohol hand sanitizer. Another before and after study with control group ${ }^{26}$ compared the $4 \%$ antiseptic solution with the introduction of a $1 \%$ antiseptic solution. Three other before and after studies with no control group ${ }^{27-29}$ introduced an alcohol hand sanitizer alone. In the remaining 2 studies in this group, one used a randomization design with a comparison group ${ }^{30}$ to compare handwashing with the use of gown and glove isolation, and the other study was a randomized equivalence trial ${ }^{31}$ comparing alcohol with antiseptic solutions.

Three studies demonstrated a decrease in infection rates with the hand sanitizer. ${ }^{21,24,25}$ Two other studies $^{22,23}$ reported no significant differences in HCAI rates between handwashing and the introduction of a hand sanitizer. Another study ${ }^{26}$ reported a decrease in MRSA rates postintervention.

One study ${ }^{29}$ reported a significant reduction in MRSA rates $(P=.03)$, another ${ }^{27}$ reported a significant reduction in MRSA $(P=.01)$ and $\operatorname{VRE}(P<.001)$ rates, and the results of another study ${ }^{28}$ were not statistically significant. One study ${ }^{30}$ showed significant reductions in infection rates before and after in each of the groups $(P=.008$ and $P=.008$, respectively), and another study $^{31}$ showed no significant differences between the 2 protocols $(P=.05)$. In assessing these 11 studies as a whole, 4 had fatal flaws, and all 4 failed to demonstrate adequate statistical analysis (Table 4).

\section{Category 3. Infection control practices}

Eight studies (25.81\%) introduced or modified infection control practices to study their impact on the 


\begin{tabular}{|c|c|c|c|c|c|c|}
\hline \multirow[b]{2}{*}{ Primary author (date) } & \multirow[b]{2}{*}{ Design } & \multirow[b]{2}{*}{ Intervention } & \multirow[b]{2}{*}{ Findings } & \multicolumn{2}{|c|}{ Scoring tool ${ }^{10}$} & \multirow[b]{2}{*}{ Comments } \\
\hline & & & & Score & e Fatal flaws & \\
\hline \multicolumn{7}{|c|}{ Category I. Multimodal hand hygiene initiatives } \\
\hline \multirow[t]{4}{*}{ Pittet $(2000)^{14}$} & $\begin{array}{l}\text { Before and after (no } \\
\text { control group) }\end{array}$ & Multimodal & Significant reduction $(P=.04)$ & 1 & II & $\begin{array}{l}\text { Uncontrolled bias or confounding factor } \\
\text { (reduction in infection rates and MRSA } \\
\text { transmission possibly confounded by } \\
\text { multiple interventions) }\end{array}$ \\
\hline & & & $\begin{array}{l}7 \% \text { decrease in overall nosocomial } \\
\text { infection }\end{array}$ & & & Other flaws: \\
\hline & & & Significant reduction $(P<.001)$ & & & $\begin{array}{l}\text { Observational bias/possibility of } \\
\text { Hawthorne effect }\end{array}$ \\
\hline & & & $\begin{array}{l}0.01 \% \text { decrease in MRSA } \\
\text { transmission rates }\end{array}$ & & & \\
\hline $\operatorname{Lam}(2004)^{15}$ & $\begin{array}{l}\text { Before and after (no } \\
\text { control group) }\end{array}$ & Multimodal & $\begin{array}{c}\text { No significant reduction }(P=.14) \\
0.51 \% \text { decrease in HCAl rate }\end{array}$ & 2 & & $\begin{array}{l}\text { Possibility that HCWs behavior changed } \\
\text { because they were being observed }\end{array}$ \\
\hline \multirow[t]{2}{*}{ Zerr $(2005)^{16}$} & $\begin{array}{l}\text { Before and after (no } \\
\text { control group) }\end{array}$ & Multimodal & Significant reduction $(P=.0 \mathrm{I})$ & 3 & & \\
\hline & & & $0.37 \%$ decrease in rotavirus rate & & & \\
\hline Aragon $(2005)^{17}$ & $\begin{array}{l}\text { Before and after (no } \\
\text { control group) }\end{array}$ & Multimodal & $\begin{array}{l}6 \text { mo: } 17 \% \text { decrease in MRSA, } 13 \% \\
\text { decrease in VRE } \\
\text { I yr: increase trend for MRSA and } \\
\text { VRE } \\
\text { No } P \text { value }\end{array}$ & 3 & & \\
\hline \multirow[t]{2}{*}{ Won $(2004)^{18}$} & $\begin{array}{l}\text { Before and after (no } \\
\text { control group) }\end{array}$ & Multimodal & Significant reduction $(P=.003)$ & 3 & & \\
\hline & & & $\begin{array}{l}0.44 \% \text { decrease in } \mathrm{HCAl} \text { rates; in } \\
\text { particular significant reduction } \\
(P=.002) \\
0.23 \% \text { decrease in respiratory tract } \\
\text { infections }\end{array}$ & & & \\
\hline \multirow[t]{2}{*}{ Hilburn $(2003)^{19}$} & $\begin{array}{l}\text { Before and after (no } \\
\text { control group) }\end{array}$ & Multimodal & $36.1 \%$ decrease in infection rates & 3 & & $\begin{array}{l}\text { No hand hygiene compliance data } \\
\text { provided }\end{array}$ \\
\hline & & & No $P$ value & & & \\
\hline \multirow[t]{7}{*}{ Johnson $(2005)^{20}$} & $\begin{array}{l}\text { Cohort study (no } \\
\text { control group) }\end{array}$ & Multimodal & Significant reduction $(P=.0 \mathrm{I})$ & 1 & II & $\begin{array}{l}\text { Uncontrolled bias or confounding factor } \\
\text { (data collection unblinded) }\end{array}$ \\
\hline & & & $57 \%$ decrease in MRSA bacteremia & & & Other flaws: \\
\hline & & & & & & $\begin{array}{l}\text { Difficult to determine the merits of each } \\
\text { intervention (simultaneous } \\
\text { interventions) }\end{array}$ \\
\hline & & & & & & $\begin{array}{l}\text { Reliance on historical controls/possibility } \\
\text { of Hawthorne effect }\end{array}$ \\
\hline & & & & & & Possible that change in MRSA \\
\hline & & & & & & $\begin{array}{l}\text { bacteremias were because of natural } \\
\text { variability over time }\end{array}$ \\
\hline & & & & & & $\begin{array}{l}\text { Only percentage reductions in MRSA } \\
\text { bacteremia and ESBL were provided }\end{array}$ \\
\hline
\end{tabular}




\begin{tabular}{|c|c|c|c|c|c|c|}
\hline \multirow[b]{2}{*}{ Primary author (date) } & \multirow[b]{2}{*}{ Design } & \multirow[b]{2}{*}{ Intervention } & \multirow[b]{2}{*}{ Findings } & \multicolumn{2}{|c|}{ Scoring tool ${ }^{10}$} & \multirow[b]{2}{*}{ Comments } \\
\hline & & & & Score & Fatal flaws & \\
\hline \multicolumn{7}{|c|}{ Category 2. Hand hygiene products } \\
\hline \multirow[t]{2}{*}{$\mathrm{Ng}(2004)^{21}$} & $\begin{array}{l}\text { Before and after (no } \\
\text { control group) }\end{array}$ & Handwashing & $\begin{array}{l}\text { Significant reduction }(P=.048), 11 \% \\
\text { fewer MRSA infections in the glove } \\
\text { AHS protocol }\end{array}$ & 3 & & \\
\hline & & vs AHS before gloves & $\begin{array}{l}0.87 \% \text { decrease of late-onset } \\
\text { systemic infection }\end{array}$ & & & \\
\hline \multirow[t]{2}{*}{ Larson $(2005)^{22}$} & $\begin{array}{l}\text { Sequential crossover study } \\
\text { (with comparison group) }\end{array}$ & $\begin{array}{l}\text { Antiseptic handwash vs alcohol } \\
\text { handrub }\end{array}$ & $\begin{array}{l}\text { No significant differences in neonatal } \\
\text { infections (any infections }[P=.88] \text {, } \\
\text { bloodstream infections }[P=.94] \text {, } \\
\text { CNS infection }[P=.68] \text {, skin } \\
\text { infection }[P=.08] \text {, pneumonia }[P= \\
\text {.37]) between the } 2 \text { groups }\end{array}$ & 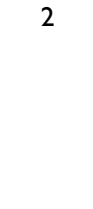 & & Self reported hand hygiene practices \\
\hline & & & & & & $\begin{array}{l}\text { Potential investigator bias (because of no } \\
\text { blinding of study products) }\end{array}$ \\
\hline \multirow[t]{2}{*}{ Mody $(2003)^{23}$} & $\begin{array}{l}\text { Cohort study (with } \\
\text { control group) }\end{array}$ & $\begin{array}{l}\text { alcohol-based handrub vs } \\
\text { handwashing only }\end{array}$ & $\begin{array}{l}\text { No differences in HCAI between the } \\
2 \text { units }\end{array}$ & 1 & I & Inadequate sample size \\
\hline & & & No $P$ value & & IV & $\begin{array}{l}\text { Inadequate statistical analysis (no power } \\
\text { calculation-to compare between } \\
\text { groups } \\
\text { Other flaws:) } \\
\text { Self-reported hand hygiene practices }\end{array}$ \\
\hline \multirow[t]{2}{*}{ Marena $(2002)^{24}$} & $\begin{array}{l}\text { Cohort crossover study } \\
\text { (with comparison group) }\end{array}$ & Plain soap vs chlorhexidine & Decrease in HCAls & 2 & & Limited sample size and follow-up period \\
\hline & & & No $P$ value & & & $\begin{array}{l}\text { Possibility that HCWs behavior changed } \\
\text { because they were being observed }\end{array}$ \\
\hline \multirow[t]{2}{*}{ Fendler $(2002)^{25}$} & $\begin{array}{l}\text { Cohort study (with } \\
\text { control group) }\end{array}$ & Hand sanitizer vs handwashing & $\begin{array}{l}\text { Significant reduction }(P<.05), 30.4 \% \\
\text { decrease in infection rates with } \\
\text { hand sanitizer }\end{array}$ & 1 & II & $\begin{array}{l}\text { Uncontrolled bias or confounding factor } \\
\text { (different level of acuity between } \\
\text { intervention vs control group) }\end{array}$ \\
\hline & & & & & IV & $\begin{array}{l}\text { Inadequate statistical analysis (groups } \\
\text { cannot be compared-no power } \\
\text { calculation performed and a difference } \\
\text { in acuity levels between the groups } \\
\text { exists) }\end{array}$ \\
\hline \multirow[t]{4}{*}{ Brittain $(2004)^{26}$} & $\begin{array}{l}\text { Before and after (with } \\
\text { control group) }\end{array}$ & $\begin{array}{l}\text { I\% antiseptic solution vs } 4 \% \\
\text { antiseptic (control group) }\end{array}$ & $\begin{array}{l}68 \% \text { lower MRSA infection rates } \\
\text { postintervention on orthopedic } \\
\text { ward }\end{array}$ & I & I & Inadequate sample size \\
\hline & & & No $P$ value & & II & $\begin{array}{l}\text { Uncontrolled bias or confounding factor } \\
\text { (no confirmation of homogeneity } \\
\text { between groups) }\end{array}$ \\
\hline & & & & & IV & $\begin{array}{l}\text { Inadequate statistical analysis (groups not } \\
\text { comparable-no power calculation } \\
\text { performed and-no confirmation of } \\
\text { homogeneity between groups) }\end{array}$ \\
\hline & & & & & & Other flaws: \\
\hline
\end{tabular}


Gordin $(2005)^{27}$
No significant change $(P=.62)$ in $C$

Alcohol hand gel and performance feedback

Handwashing vs gown and glove isolation comparison group)

Parienti $(2004)^{31}$

Randomized equivalence trial

Hand rubbing (alcohol) vs hand scrubbing (antiseptic)

\section{Category 3. Infection control practices}

Schelenz $(2005)^{34} \quad$ Before and after (no control group) Infection control practices

$\operatorname{Kac}(2000)^{35}$

Before and after (no control group) Infection control practices

Significant reduction $(P=.0008)$ in
Significant reduction $(P=.01)$

$21 \%$ decrease in MRSA

Significant reduction $(P<.00 I)$

$41 \%$ decrease in VRE difficile and rates

No differences

No $P$ value

Compliance with handwashing not monitored

Clear and concise study

No data on extent of use of product or hand hygiene compliance

Inadequate sample size (study underpowered)

Uncontrolled bias or confounding factor (unclear participant inclusion criteria and data collection unblinded)

Unclear operational definitions (unclear definition of outcome)

IV No statistical analysis

Significant reduction $(P=.03)$

Unclear whether rates were infection or colonization

$0.9 \%$ reduction in MRSA rates gown and glove isolation group

$2.6 \%$ decrease in infection rate

Significant reduction $(P=.008)$ in

handwashing group

$1.9 \%$ decrease in infection rate

No significant differences between the 2 protocols $(P=.05)$

A $0.04 \%$ difference between the 2 protocols

Significant reduction $(P=.003)$

$2.14 \%$ drop in MRSA nosocomial infection/colonization Significant reduction $(P=.014)$

$0.89 \%$ drop in bloodstream MRSA infection

Significant reduction $(P=.02)$

$6.8 \%$ decrease in MRSA infection

rates between the pre- and 1994 postintervention

Significant reduction $(P=.002)$

$8.1 \%$ decrease in MRSA infection

rates between pre- and 1996

postintervention

\section{Limited sample size}

First randomized trial to compare hand rubbing with alcohol vs traditional hand scrubbing in the routine surgical setting

No mention of institutional review board approval

Unsure about which intervention was critical for success

Use of historical controls

Difficult to determine the merits of each intervention 


\begin{tabular}{llr}
\hline Primary author (date) & Design & Intervention \\
\hline Lai $(1998)^{32}$ & Before and after (no control group) & Infection control practices \\
Schelonka $(2005)^{36}$ & Before and after (no control group) & Infection control practices
\end{tabular}

Zafar $(1998)^{33}$

Before and after (no control group) Infection control practices

Kurlat $(1998)^{37}$

Before and after (no control group) Infection control practices

Andersen $(2005)^{38}$

Before and after (with Before group Infection control practices acting as control)

Makris $(2000)^{39}$

Before and after (with control group) Infection control practice

\section{Category 4. Infection control policies}

Stone $(1998)^{40} \quad$ Before and after (no control group) Infection control policies

\section{Scoring tool ${ }^{10}$}

Score Fatal flaws

\section{Comments}

Overall decrease in VRE rates

No $P$ value

Significant reduction $(P=.002)$

Year I: $26 \%$ reduction in infection rate

Significant reduction $(P<.001)$

Year 2 and 3: $29 \%$ reduction

Significant reduction $(P<.05)$

$60 \%$ decrease in $C$ difficile

Significant reduction $(P<.003)$

$76 \%$ decrease in bacteremia rate

Significant reduction $(P=.05)$

$12 \%$ decrease of bloodstream infections

$0.24 \%$ decrease in overall infection No $P$ value

No significant reduction $(P=.06)$ of upper respiratory infections

Significant reduction $(P<.05)$

$1.4 \%$ decrease in $C$ difficile and Significant reduction $(P<.01)$ $2.01 \%$ decrease in MRSA
Inadequate statistical analysis

Other flaws:

No hand hygiene intervention described Compliance with all individual practices not measured

Impossible to determine role of hand hygiene in this multiintervention study

Uncontrolled bias or confounding factor (data collection unblinded) Inadequate statistical analysis Other flaws:

No examination of hand hygiene per se Role of $\mathrm{HH}$ can not be determined exactly because of multiple interventions, although most of the interventions were aimed at contact using clean hands

Inadequate sample size (study underpowered)

Actual sample size was too small: 174 infants instead of 220 required

Other flaws:

Impossible to have determined the role of hand hygiene even if a statistical significant outcome differences had been noted

Adherence to all the interventions was not assessed

Inadequate sample size

Uncontrolled bias or confounding factor (groups not comparable-no power calculation)

Other flaws:

Role of hand hygiene cannot be determined

Unclear what contribution hand hygiene made over other interventions 
Presence of alcohol hand gel was associated with a decreased risk gastrointestinal infections (odd ratio, 0.66)

$10 \%$ reduction of HCAls in phase and $40 \%$ reduction of HCAls in phase III

No $P$ value

Overall incidence rate of hospitalacquired diarrhea, $3.6 \%$

No $P$ value

Larson $(2000)^{44}$

Before and after (with control group) Top level administration intervention Significant reduction $(P=.002)$ in

VRE rates for intervention hospita

(RR, .29)

No significant change $(P=.25)$ in

MRSA rates
Unclear operational definitions (unclear definition of outcome)

Outcome could actually be communityacquired infections rather than HAI

Other flaws:

Survey questions were about the presence of policies and not about a measure of current practice

Sample size not large enough to detect significant change in infection rates

Small sample size

Participation was on a voluntary basis Self-assessment questionnaires

Duration of observation not very long, and VRE rates can vary substantially over time without intervention naturally

$A H S$, alcohol hand sanitizer; ESBL, extended-spectrum $\beta$-lactamase. 
reduction of HCAIs. Six studies were before and after studies with no control group, ${ }^{32-37}$ and 2 studies $^{38,39}$ had a control group.

Two of the studies, 1 with a control group ${ }^{39}$ and 1 without a control group, ${ }^{36}$ reported a decrease in overall HCAI rates, but it was not possible to determine the role of hand hygiene in any of these studies. One study that used a before group as a control group ${ }^{38}$ reported a significant reduction $(P=.05)$ of bloodstream infections. In the remaining studies without a comparison control, one ${ }^{37}$ reported a significant reduction $(P<.003)$ in the bacteremia rate; two ${ }^{34,36}$ reported a significant reduction $(P=.003$ and $P=.002$, respectively) in MRSA rates; one ${ }^{32}$ reported a decrease in VRE rates; and one ${ }^{33}$ reported a significant decrease $(P<.05)$ in $C$ difficile. In addition to the lack of clarity on the role of hand hygiene in all of these studies, 4 out of 8 studies exhibited at least 1 fatal design flaw (Table 4).

\section{Category 4. Infection control policies}

Two studies $(6.45 \%)$ introduced an infection control policy to study its impact on the reduction of HCAIs. One study, ${ }^{40}$ a before and after design with no control group, reported a significant reduction in both $C$ difficile $(P<.05)$ and MRSA $(P<.01)$. The other study, ${ }^{41}$ a cross-sectional design with no control group that reported a significant reduction $(P<.01)$ in the risk of hospital-associated gastrointestinal infections, demonstrated 2 fatal flaws including an unclear definition of outcome and the presence of confounding factors.

\section{Category 5. Other interventions}

Three studies $(9.68 \%)$ introduced organizational interventions to study their impact on the reduction of HCAIs. In a 2004 before and after study with no control group by Swoboda et $\mathrm{al}^{42}$ an electronic monitoring system was implemented to improve hand hygiene and decrease HCAIs, but the results were not statistically significant. In Jusot et al's 2003 cohort study with no control group, ${ }^{43}$ neonatal unit staff were surveyed to investigate the relationship between hygienic measures and the overall incidence rate of hospital-acquired diarrhea, but the results were not statistically significant.

In another study, Larson et $\mathrm{al}^{44}$ used a before and after design with control group to examine the relationship between a top level administration intervention aimed at changing organizational culture and hospital VRE and MRSA rates. No significant change $(P=.25)$ in MRSA rates were found, but VRE rates decreased significantly $(P=.002)$ for the intervention hospital for the duration of the study, which was over a period of 8 months. The caution with these findings is that VRE rates can naturally vary substantially over time. Nonetheless, the study design ensured that the link between hand hygiene as measured by soap utilization per patient-day and HCAI rates was demonstrably clear. One study ${ }^{43}$ received a score of 2 , and the other 2 studies $^{42,44}$ received a score of 3 (Table 4).

\section{Limitations}

The limitations of this integrative review were (1) that the search only included articles that had the term hand hygiene or handwashing in their title as the focus of the paper because of the large volume of publications available in this area and (2) that the selection of articles was limited to English and to studies conducted in developed countries to allow for better comparison of the results.

\section{DISCUSSION}

Overall, the results of the studies, given the frequent limitations to their rigor, do not demonstrate a strong relationship between hand hygiene interventions and decreased incidence of HCAIs. Furthermore, the multiple interventions used and the diverse factors related to the presence of HCAIs make it difficult or impossible to isolate the specific effects of hand hygiene or of any other component of the interventions. In addition, there is a paucity of theoretically driven research approaches, which makes it very difficult to weigh the relative merits of the particular HHI tested in each study in relation to the evidence that has accumulated to date. As Sales et al note, researchers need to be able to determine and evaluate the theoretic assumptions that underlie specific interventions if they are to formulate more coherent and sufficient rationale for their choices in strategies, interventions, tools, and study design. ${ }^{45}$ Equally, reviewers of research, policy makers, and practitioners all need to understand the theoretic rationale for specific interventions if they are to make informed choices about which intervention might work best for their settings, under which conditions, and at what possible cost.

The most frequent methodologies currently used in this research area are ecologic studies or before and after studies. These types of study designs limit generalizability because there are no way to control for natural variation and random chance. Without an independent control group that is equivalent to the study group in all other respects, it is possible that the observed differences are simply because of random chance or natural variability in the infection rates over the period of observations.

Ultimately, "the choice of design [is] dependent upon the purpose of the evaluation and the degree 
of control the researchers have over the delivery of the intervention(s)." ${ }^{46}$ Although a randomized controlled trial is the most robust study design, it is challenging to carry it out in this field for several reasons. For example, the use of control groups, in light of expert recommendations about hand hygiene, may raise questions about clinical equipoise. There can also be prohibitive costs associated with conducting a randomized control trial in which the unit of analysis must be at minimum at the level of a hospital unit, and preferably at the hospital level, because of the possibility of communication between units. In addition, the blinding of patients, practitioners, or investigators to a hand hygiene regimen is challenging, with the introduction of bias or confounding factors a real possibility. Furthermore, adequately powered sample sizes are not easy to obtain. When the number of additional factors that influence the comparability of one setting to another are considered, including but not limited to variability in organizational policies, staffing practices, patient characteristics, migrating equipment and facility design, the task of finding valid comparison controls can discourage the most determined researcher.

\section{Characteristics of a well-designed hand hygiene intervention study}

Overall, the current studies present inconsistent parameters in their design and thus make it difficult to accurately interpret and compare the research findings in this body of literature. One of our recommendations is to use a modified version of Larson's recommendations for this area of research ${ }^{10}$ to establish some common characteristics for future studies. In our estimation, these characteristics should include the following:

- Adequately powered sample size determined in advance to support conclusions;

- use of either (1) a pre- and post-with-comparison control design or (2) a cluster randomized controlled trial such as the recent trial of a multifaceted intervention to reduce antimicrobial prescriptions in nursing homes ${ }^{47}$;

- a detailed outline of the features of experimental settings and their control counterparts;

- baseline measurement of HCAIs rates with a minimum number of observations as dictated by proper sample size calculation and at least 6 to 12 months of data;

- clear identification of possible confounding variables;

- identification of a consistent hand hygiene audit tool and observation method;

- clear statement of inclusion and exclusion criteria;

- blinded data collection;
- clear and detailed description of interventions;

- clearly listed operational definitions;

- appropriate and clearly identified statistical analysis;

- evidence that the intervention was actually implemented (ie, quality control);

- common definition of outcomes (ie, HCAIs rates); and

- an explicit theoretic framework for the study that (1) clearly articulates the evidence to date on the relationship between HHIs and HCAIs and that (2) links the assessment of the evidence with sound rationale for the intervention developed and the overall research design.

Even though it is highly unlikely that researchers would consistently meet all of these criteria, the explicit use of this template to guide the design and dissemination of intervention research would enable both scientists and their target audiences to transparently identify and evaluate the limitations of specific studies in relation to chosen study approaches. ${ }^{45,48,49}$ As the field progresses, the theoretic and methodologic deficits that repeatedly surface should guide us to generate better intervention theories and more rigorous research designs.

From evidence to uptake: Exploring additional research approaches. Grimshaw et al have already noted that "some strategies [are] not amenable to randomization" in health systems research ${ }^{50}$ In the field of ecosystems management, ecologist Crawford "Buzz" Holling and Gunderson have extended this observation to argue that 2 forms of science are needed to adequately understand and manage the complex socioecologic systems that characterize today's technologic world. $^{51-53}$ In essence, Hollings and others who share a "two cultures of science" perspective equally value an analytic "science of parts" and an integrative "systems science of the whole." 52 In the first analytic line of scientific inquiry, researchers pursue vital questions in ecology, biology, microbiology, and other fields to understand how various elements of the world interact under specific controlled experimental conditions. In the second integrative line of science, researchers ask the following: Now that we better understand how various parts of the world work in an experimental mode, what system conditions are needed for people, communities, and institutions to use evidence about the world to effectively adapt and change? With these latter systems science questions, we are using research to discover how we can translate what we learn from controlled experiments into the effective study and management of an uncontrollable world.

We propose that equal attention to both the analytic and integrative sciences are as important for sound health systems management as they are for effective ecosystems management. If we are right, we need at 
least 2 research approaches to accelerate the conduct and uptake of research that leads us to reliable, enduring reductions in preventable HCAIs. For example, we know that, in present-day infection control practice, multiple interventions are used to resolve real day-today outbreaks as rapidly as we can muster a response. However, we also know that, in our understandable urgency to act without strong levels of evidence, we may be allocating significant resources to interventions that add no or little value or that could even cause unanticipated harms. In a 2-science world, we suspect that an adequate way forward from this state of affairs is distinguished by at least 2 parallel paths of concurrent action. First, in terms of analytic science, we may want to conduct a select number of focused studies that examine specific hand hygiene interventions (such as education, gowns, gloves, cleaning methods, and products) one by one in isolation to determine the relative impact of various interventions on specific HCAIs one at a time. These studies would explore the biologic gradients between hand hygiene and particular HCAIs to establish whether the results are or are not the same for each infection. We should also seek to conduct future studies of complex, multilayered HHIs to determine which synergistic combinations of interventions are most effective and affordable in reducing what HCAIs and under what conditions. Furthermore, as we have already noted, sound HHI studies of any kind should incorporate a clearly articulated and justified theoretic rationale, the use of comparable control groups, and other features of a robust analytic design. In addition, we will need ongoing systematic reviews in the Cochrane tradition of the analytic scientific evidence that accumulates to provide researchers with clear critiques of the evidence and necessary insights into the methodologic challenges with this kind of research.

As we accumulate empirical evidence on which individual and multilayered HHIs are most effective under what controlled experimental conditions, though, we predict that many of the methodologic challenges to conducting rigorous controlled trials of infection control programs such as multifaceted HHIs will prevail. Accordingly, we suggest that there is a pressing need to develop concurrently a parallel, critical path of integrative science for multifaceted HHIs because such programs actually constitute multilayered organizational interventions in complex (and therefore continually confounding) health care systems that are characterized by instability and change. ${ }^{12}$ Here, we need to use a range of theoretically driven questions about preventing, minimizing, and managing real outbreaks in a real, inevitably uncontrolled world. In this integrative line of scientific inquiry, our "systems science" question would be as follows: What system conditions enable people, communities, and organizations to effectively translate and adapt evidence-informed infection control policies and practices to their specific, local, real worlds of health care $?^{54}$ In the case of multifaceted HHIs, we would then study the adaptation of evidence-informed HHI guidelines to determine what helps or hinders us from achieving both ongoing, affordable reductions to a number of HCAIs in a range of health care settings and earlier detection and control of emerging threats.

To reach these goals of integrative science, researchers need to become familiar with a range of mixed methods that enable us to understand health systems change from a variety of qualitative and quantitative vantage points, including but not limited to such research approaches as retrospective, real-time, and planned prospective case studies; institutional ethnography; participatory action research; and other designs. ${ }^{51}$ In addition, to ensure that we match the necessary questions about systems change with coherent theories and defensible methods, we agree with safety researchers who urge us to reach beyond a sole focus on evidence-based medicine to a broader and more interdisciplinary view of safety science that encompasses not only the health disciplines but a variety of experts across the social, engineering, economic, political, and ecologic sciences. ${ }^{55-59}$ For that matter, ongoing dialogue with experts in many of these fields would arguably strengthen both our analytic and integrative research designs in many areas of health systems research. Furthermore, to synthesize the findings across both categories of science into meaningful guidance for decision makers and practitioners, we will need what Pawson et al call realist reviews of research. ${ }^{12}$ In this alternate method of systematic review, the contention is that "complex policy interventions" should be informed by syntheses of research across qualitative and quantitative methods that contextualize findings into theoretically driven, principles-based recommendations for local contextualization rather than universal rules of treatment based on effect sizes. ${ }^{12}$ According to Pawson et al, a good realist review "learns from (rather than controls for) real-world phenomena such as diversity, change, idiosyncrasy, adaptation, cross contamination, and programme failure." 12 In other words, we not only need more good science, but at least 2 kinds of science, and at least two kinds of critical review methods, to synthesize our findings in infection control research into practical knowledge that we can readily use.

\section{CONCLUSION}

Even though the number of publications on HHIs is impressively large, the methodologic issues for 
this area of research remain significant. This integrative review demonstrates that the evidence linking hand hygiene interventions and HCAIs is present, but it is not well quantified. It is clear that we need more robust analytic research designs to obtain better evidence on which HHIs are associated with reduced incidences of what HCAIs in a variety of settings. From our perspective, it is equally clear that, even as we gather this evidence, we also face challenging questions of systems science where we need to integrate evidence on effective HHIs with better knowledge of what drives human, organizational, and health system policy and practice toward evidence-informed, sustainable infection prevention and control.

It is very unlikely that we will ever eliminate infections entirely, even if we achieved $100 \%$ compliance with stringent hand hygiene. It is more likely that, for HHIs as well as for other infection control interventions, we may initially see a steep improvement as did Semmelweis, ${ }^{60}$ and yet still find that controlling the last $5 \%$ of infections requires $90 \%$ of our efforts. The development of a more resourceful, evidence-based approach to infection control research and practice therefore remains a very complex yet vital challenge for modern health systems that science will not solve anytime soon. However, if we initiate ongoing dialogue with colleagues across a much wider landscape of expertise than we have previously traveled, we may find that we not only generate better evidence on the relationship between various HHIs and HCAIs but we also create better strategies for translating evidence into wiser resource allocation and the reduction of preventable harms. In the meantime, we can only hope that more and more organizations and practitioners will not let evidence-based medicine act as an erroneous "intellectual hegemony",51 that substitutes for sound judgment and that, instead of waiting for irrefutable analytic scientific evidence, a rising community of the conscientious will insist that we all consistently and thoroughly wash our hands.

The authors thank the Canadian Patient Safety Institute for their support in this initiative and Orvie Dingwall, the librarian and information specialist at the Canadian Patient Safety Institute, for her extensive help and assistance with the literature search and strategy.

\section{References}

I. Baker GR, Norton PG, Flintoft V. The Canadian Adverse Events Study: the incidence of adverse events among hospital patients in Canada. CMAJ 2004; 170:1678-86.

2. Zoutman DE, Ford D, Bryce E, Gourdeau M, Hebert G, Henderson E, et al. The state of infection surveillance and control in Canadian acute care hospitals. Am J Infect Control 2003;31:266-73.

3. Burke JP. Infection control: a problem for patient safety. N Engl J Med 2003;348:65I-6.
4. Larson EL. APIC Guideline for handwashing and hand antisepsis in health care settings. Am J Infect Control 1995;23:251-69.

5. Boyce JM, Pittet D. Guideline for hand hygiene in health-care settings: recommendations of the health care infection control practices advisory committee and the HICPAC/SHEA/APIC/IDSA Hand Hygiene Task Force. Infect Control Hosp Epidemiol 2002;23: I-48.

6. Pittet D. Improving compliance with hand hygiene in hospitals. Infect Control Hosp Epidemiol 2000;21:38I-6.

7. Suresh G, Cahill J. National patient safety goals. How "user friendly" is the hospital for practicing hand hygiene? An ergonomic evaluation. Joint Commission on Accreditation of Healthcare Organizations 2007;33:17I-8.

8. Larson E. A causal link between handwashing and risk of infection? Examination of the evidence. Infect Control Hosp Epidemiol 1988;9: 28-36.

9. Larson E. Skin hygiene and infection prevention: more of the same or different approaches? Clin Infect Dis 1999;29:1287-94.

10. Larson E. State-of-the-science-2004: time for a "No Excuses/No Tolerance" (NET) strategy. Am J Infect Control 2005;33:548-57.

II. Silvestri L, Petros AJ, Sarginson RE, et al. Handwashing in the intensive care unit: a big measure with modest effects. J Hosp Infect 2005;59: 172-9.

12. Pawson R, Greenhalgh T, Harvey G, et al. Realist review: a new method of systematic review designed for complex policy interventions. J Health Serv Res Policy 2005; 10:21-34.

13. Garner JS, Jarvis WR, Emori TG, et al. CDC definitions for nosocomial infections. In: Olmsted RN, editor. APIC infection control and applied epidemiology: principles and practice. St Louis: Mosby; 1996. p. AI-A20.

14. Pittet D, Hugonnet S, Harbarth S, et al. Effectiveness of a hospital-wide programme to improve compliance with hand hygiene. Lancet 2000; 356:1307-12.

15. Lam BC, Lee J, Lau YL. Hand hygiene practices in a neonatal intensive care unit: a multimodal intervention and impact on nosocomial infection. Pediatrics 2004; I I4:e565-7I.

16. Zerr DM, Allpress AL, Heath J, et al. Decreasing hospital-associated rotavirus infection: a multidisciplinary hand hygiene campaign in a children's hospital. Pediatr Infect Dis J 2005;24:397-403.

17. Aragon D, Sole ML, Brown S. Outcomes of an infection prevention project focusing on hand hygiene and isolation practices. AACN Clin Issues 2005;16:121-32.

18. Won SP, Chou HC, Hsieh WS, et al. Handwashing program for the prevention of nosocomial infections in a neonatal intensive care unit. Infect Control Hosp Epidemiol 2004;25:742-6.

19. Hilburn J, Hammond BS, Fendler EJ, et al. Use of alcohol hand sanitizer as an infection control strategy in an acute care facility. Am J Infect Control 2003;31:109-16.

20. Johnson PD, Martin R, Burrell LJ, et al. Efficacy of an alcohol/chlorhexidine hand hygiene program in a hospital with high rates of nosocomial methicillin-resistant Staphylococcus aureus (MRSA) infection. Med J Aust 2005;183:509-14.

21. Ng PC, Wong HL, Lyon DJ, et al. Combined use of alcohol hand rub and gloves reduces the incidence of late onset infection in very low birth weight infants. Arch Dis Child Fetal Neonatal Ed 2004;89: F336-40.

22. Larson EL, Cimiotti J, Haas J, et al. Effect of antiseptic handwashing vs alcohol sanitizer on health care-associated infections in neonatal intensive care units. Arch Pediatr Adolesc Med 2005;159: 377-83.

23. Mody L, McNeil SA, Sun R, et al. Introduction of a waterless alcoholbased hand rub in a long-term-care facility. Infect Control Hosp Epidemiol 2003;24:165-7I.

24. Marena C, Lodola L, Zecca M, et al. Assessment of handwashing practices with chemical and microbiologic methods: preliminary results from a prospective crossover study. Am J Infect Control 2002;30: 334-40. 
25. Fendler EJ, Ali Y, Hammond BS, et al. The impact of alcohol hand sanitizer use on infection rates in an extended care facility. Am J Infect Control 2002;30:226-33.

26. Brittain B. MRSA control: a simple approach using triclosan antiseptic wash. Aust Infect Control 2004;9:65-8.

27. Gordin FM, Schultz ME, Huber RA, et al. Reduction in nosocomial transmission of drug-resistant bacteria after introduction of an alcohol-based handrub. Infect Control Hosp Epidemiol 2005;26:650-3.

28. King S. Provision of alcohol hand rub at the hospital bedside: a case study. J Hosp Infect 2004;56(Suppl 2):SI0-2.

29. MacDonald A, Dinah F, MacKenzie D, et al. Performance feedback of hand hygiene, using alcohol gel as the skin decontaminant, reduces the number of inpatients newly affected by MRSA and antibiotic costs. J Hosp Infect 2004;56:56-63.

30. Slota M, Green M, Farley A, et al. The role of gown and glove isolation and strict handwashing in the reduction of nosocomial infection in children with solid organ transplantation. Crit Care Med 2001;29: 405-12.

3I. Parienti JJ, Thibon P, Heller R, et al. Hand-rubbing with an aqueous alcoholic solution vs traditional surgical hand-scrubbing and 30-day surgical site infection rates: a randomized equivalence study. JAMA 2002; 288:722-7.

32. Lai KK, Kelley AL, Melvin ZS, et al. Failure to eradicate vancomycin-resistant enterococci in a university hospital and the cost of barrier precautions. Infect Control Hosp Epidemiol 1998;19:647-52.

33. Zafar $A B$, Gaydos LA, Furlong WB, et al. Effectiveness of infection control program in controlling nosocomial Clostridium difficile. Am J Infect Control 1998;26:588-93.

34. Schelenz S, Tucker D, Georgeu C, et al. Significant reduction of endemic MRSA acquisition and infection in cardiothoracic patients by means of an enhanced targeted infection control programme. J Hosp Infect 2005;60: 104-10.

35. Kac G, Buu-Hoi A, Herisson E, et al. Methicillin-resistant Staphylococcus aureus: nosocomial acquisition and carrier state in a wound care center. Arch Dermatol 2000;136:735-9.

36. Schelonka RL, Scruggs S, Nichols K, et al. Sustained reductions in neonatal nosocomial infection rates following a comprehensive infection control intervention. J Perinatol 2006;26:176-9.

37. Kurlat I, Corral G, Oliveira F, et al. Infection control strategies in a neonatal intensive care unit in Argentina. J Hosp Infect 1998;40:149-54.

38. Andersen C, Hart J, Vemgal P, et al. Prospective evaluation of a multifactorial prevention strategy on the impact of nosocomial infection in very-low-birthweight infants. J Hosp Infect 2005;61:162-7.

39. Makris AT, Morgan L, Gaber DJ, et al. Effect of a comprehensive infection control program on the incidence of infections in long-term care facilities. Am J Infect Control 2000;28:3-7.

40. Stone SP, Beric V, Quick A, et al. The effect of an enhanced infectioncontrol policy on the incidence of Clostridium difficile infection and methicillin-resistant Staphyloccocus aureus colonization in acute elderly medical patients. Age Ageing 1998;27:561-8.

4I. Zerr DM, Garrison MM, Allpress AL, et al. Infection control policies and hospital-associated infections among surgical patients: variability and associations in a multicenter pediatric setting. Pediatrics 2005; II5:e387-92.
42. Swoboda SM, Earsing K, Strauss K, et al. Electronic monitoring and voice prompts improve hand hygiene and decrease nosocomial infections in an intermediate care unit. Crit Care Med 2994;32:358-63.

43. Jusot J-F, Vanhems $P$, Benzait F, et al. Reported measures of hygiene and incidence rates for hospital-acquired diarrhea in 31 French pediatric wards: is there any relationship? Infect Control Hosp Epidemiol 2003;24:520-5.

44. Larson EL, Early E, Cloonan P, et al. An organizational climate intervention associated with increased handwashing and decreased nosocomial infections. Behav Med 2000;26:14-22.

45. Sales A, Smith J, Curran G, et al. Models, strategies, and tools: theory in implementing evidence-based findings into health care practice. J Gen Intern Med 2006;21:s43-9.

46. Eccles M, Grimshaw J, Campbell M, et al. Research designs for studies evaluating the effectiveness of change and improvement strategies. Qual Safety Health Care 2003; 12:47-52.

47. Loeb M, Brazil K, lohfield L, et al. Effect of a multifaceted intervention on number of antimicrobial prescriptions for suspected urinary tract infections in residents of nursing homes: cluster randomised controlled trial. Br Med J 2005; 10:1-5.

48. Marck PB. Theorizing about systems: an ecological task for patient safety research. Clin Nurs Res 2005; 14:103-8.

49. Shojania KG, Duncan BW, McDonald KM, et al. Safe but sound: patient safety meets evidence-based medicine. J Am Med Assoc 2002;288: 508-13.

50. Grimshaw J, McAuley LM, Bero LA, et al. Systematic reviews of the effectiveness of quality improvement strategies and programmes. Qual Safety Health Care 2003; 12:298-303.

5I. Gunderson LH, Holling CS. Barriers and bridges to the renewal of ecosystems and institutions. New York: Columbia University Press; 1995.

52. Holling CS. Two cultures of ecology. Conservation Ecol 1998;2: I-6.

53. Gunderson L, Holling CS. In memory of mavericks. Conservation Ecol 2002;6: I- 10

54. Edwards N, Marck P, Virani T, et al. Whole systems change in health care: implications for evidence-informed nursing service delivery models. Ottawa: University of Ottawa; 2007.

55. Berwick DM. Broadening the view of evidence-based medicine. Qual Safety Healthcare 2005; 14:3 I5-6.

56. Wears RL. Organisations and safety in health care. Qual Safety Healthcare 2004; I3:iil.

57. Waldvogel FA. Infectious diseases in the 2 Ist century: old challenges and new opportunities. Int J Infect Dis 2004;8:5-12.

58. Marck PB, Higgs ES, Edwards N, et al. Generating adaptive health systems: an emerging framework of research and restoration for a safer world. Social Science and Humanities Research Council Working Paper Series: Paper No. I 2006. Available at: http://www. nursing.ualberta.ca/SaferSystems/projects.htm. Accessed July 31, 2007.

59. Marck PB. Field notes from research and restoration in the backcountry of modern health care. Can J Nurs Res 2006;38: I I-23.

60. Best M, Neuhauser D. Ignaz Semmelweis and the birth of infection control. Br Med J 2004; 13:233-4. 